\title{
A Review for the Adopted Techniques in Low SNR Communication Systems
}

\author{
Dhafer R. Zaghar ${ }^{1}$, Hadeel N. Abdullah², Ikhlas M. Farhan ${ }^{3}$

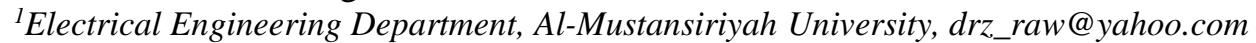 \\ ${ }^{2}$ Electrical Engineering Department, University of Technology, 30002@uotechnology.edu.iq \\ ${ }^{3}$ Electrical Engineering Department, University of Technology, 30152@uotechnology.edu.iq
}

\begin{abstract}
The study and evaluation of digital communication systems in low signal-to-noise power ratio (LSNR) environments is a hot and important topic due to its wide contemporary applications. This review is an attempt to focus on the most distinctive vital parameters required in the analysis and design of such systems. It includes a brief literature survey for many algorithms, techniques, methodologies, and mitigations that have been used to overcome the high noise problem encountered in these systems in order to guarantee reliable communication. Amongst these topics, the study reviews the most widely utilized modulation techniques in LSNR communication systems such as the On-Off Keying (OOK) and binary PSK schemes. The capacity for both AWGN and wireless fading channels is also discussed along with a clarification of the minimum limits in these types of channels.

On the other hand, some important and recommended detection and estimation techniques for low power communication systems are briefly covered. This unified study has additionally discussed spectral efficiency and bit energy tradeoff within the low-power, and broadband systems, and different limitations are stated clearly. Finally, the survey throughout this paper recommends some significant techniques that can be used in the analysis and design of successful communication systems in LSNR regimes.
\end{abstract}

Index Terms - communication systems, low SNR, low power, modulation techniques, $A W G N$.

\section{INTRODUCTION}

Communication systems can generally be classified as either bandwidth-limited or power-limited systems. In bandwidth-limited systems, spectrally-efficient modulation techniques can be adopted to save bandwidth at the expense of power whereas in powerlimited systems, power-efficient modulation techniques are utilized to save power at the expense of wider bandwidth. In systems that are both bandwidth-limited and power-limited, error-correction coding may be used to conserve power or to enhance error performance at the expense of bandwidth [1].

In digital communication systems, the quality of information transmitted from the transmitter to the receiver can be measured by the bit error rate (BER) at the receiver side. Several information transfer applications need a minimum data rate, where moderate quantity of data transferred from the source to the sink is to be achieved within a specific period of time. Thus, bandwidth minimization is a very important feature of any data communication system. When designing a digital communication system, it is necessary to understand the bounds of the channel capacity which should not be exceeded in terms of the minimum permissible signal to noise ratio (SNR) and maximum allowable bandwidth. Consequently, several digital communication system designers use the thought of channel 
capacity to mathematically confirm these limits [2]. A simple block diagram of a digital communication system is shown in Fig. 1

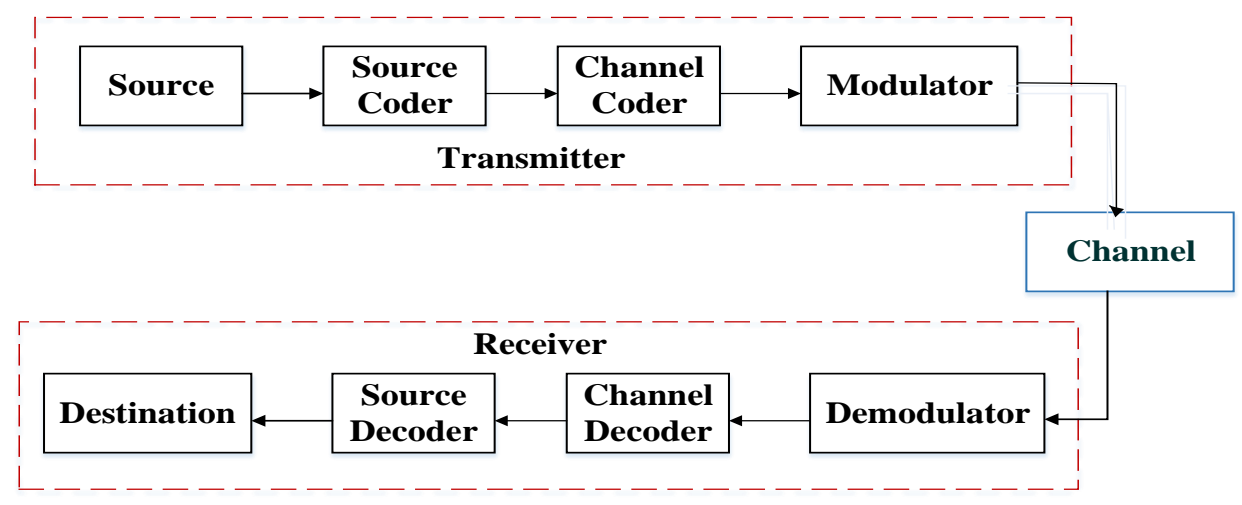

Fig.1. Block DiAgRAM OF A Digital COMMUNiCATION SYSTEM [1]

Therefore, the use of large bandwidth together with low power signals will lead to low signal to noise ratio (LSNR), making the system finite in power with respect to noise. However, it is important to study the performance of communications system in the low power regime (LPR).

In modern communications systems, the transmitted signals are often sent under high noise environment, causing the detection process of the information more difficult as a result of increased interference of the signal in the complex channel [3]. Many communication systems have high levels of noise in their environment such as light fidelity (Li-Fi) [4], [5], power line communications (PLC) [6], and under-water communications [7]. Other high interference systems include mobile communications, wireless ad-hoc networks, and ultrawide band networks which need efficient receivers that may work at very low levels of signal to noise ratio [8],[9]. However, achieving maximum energy efficiency is essential for many applications in wireless communication techniques such as massive multiple-input and multiple-output (MIMO) [10], micro-seismic monitoring [11], ultra wideband (UWB) systems [12], and ultra-low power wireless sensor networks (WSN) [13].

This work presents a brief survey for different modulation techniques and detection algorithms available in the literature. This includes a comprehensive analysis and comparative study of different techniques which have been used for communication under low SNR conditions. The organization of this review paper is as follows: section II introduces the most widely used modulation techniques in LSNR systems. Section III is devoted to investigate the detection of signals in LSNR channels. Section IV shows channel modeling, while section $\mathrm{V}$ introduces the most important LSNR estimation techniques. Section VI introduces LSNR coding techniques, then section VII introduces the spectral and the energy efficiency, and finally section VIII gives the main conclusions behind this study.

\section{MODULATION TECHNIQUES FOR LSNR SYSTEMS}

Low power modulators are more efficient for wireless communication systems. Various modulation techniques employed in LPR systems, like phase shift keying (PSK), pulse position modulation (PPM), and on-off keying (OOK) [14] are considered as low power schemes. When the communication technique is limited power such as wireless sensor 
networks, the general tendency is to choose a simple modulation scheme like OOK with low bandwidth efficiency to meet the LSNR requirements for lower bit error rates [13].

However, PPM can be considered as a special and natural perception of on-off keying. It is also demonstrated that non-coherent PPM is a promising technology to achieve wireless practical designed in ultra-low power (ULP) systems. The maximum limit of Shannon's channel capacity can be completed via the PPM with a hard decision, and the same result is provided by non-coherent binary input binary output (BIBO) channel if the threshold is selected correctly [15]. For example, the author in [16] Shows that quaternary PSK (QPSK) transmitted via Additive white Gaussian noise (AWGN) or the coherent fading in channels are effectively optimized in LSNR system, Achieve a minimal energy of bit is $-1.59 \mathrm{~dB}$.

P. Zhang et al in [15] demonstrated that modulation schemes such as BPSK, which are typically used in LSNR, can not be reliably used due to phase noise. As a result of the great balance via the performance and the complexity, the OOK modulation becomes an interesting candidate for low rate and highreliability solution when designing ULP wireless sensor networks.

E. Leitinger presented a successful model for channel capacity and the corresponding input distribution of the energy detector designed with a Gaussian approximation model [17]. Hence, OOK has become the optimal configuration scheme in LSNR communication systems.

In [18], a deep analysis is presented for BPSK-modulated random data without any pilot tones or training sequences used in initial transmissions.

Zhang, W. and Laneman, J.N. in [19] proposed a design for exploiting the spatially correlated MIMO antennas that will increase the rate of multiplication gain and result in a reduction of peak power. This design uses OOK as a simple modulation technique with hard-decision detection. However, this scheme which is especially appropriate for noncoherent LSNR communication systems, also needs low cost and low complexity solutions. As a result, The OOK modulation has latterly experienced a revived research interests, particularly from the green communication perspective of WSN supported by ULP wireless design.

It is necessary to state that ULP ideas play an important role in the design of independent power systems, as body area networks. In this systems, wireless sensor contract is supposed to be driven by energy scavenging, that effect in a primary energy budgeting. Therefore, forced at LPR, independent wireless systems generally run in LSNR environments [15].

\section{DETECTION OF SIGNALS IN A LOW POWER REGIME}

The detection of weak signals is via AWGN and the fading channels with hard and softdetection. Also, analysis of channel capacity for the coherent and non-coherent detection in the LPR regime is presented in this work. Despite the fact that the soft detection provides better realization, the hard decision is chosen when reducing the computation load [20]. Such a demand, as an example, is also imposed in sensing element networks [21].

Kramer, G. et al in [22] introduced a detailed analysis of the spectral efficiency for PSK and differential PSK (DPSK) via hard and soft detection. Also, they demonstrated that when using a hard coherent detection, the system can not send signals through the communication channel when the the energy per bit to noise power spectral density ratio (Eb/ No) is less than $0.37 \mathrm{~dB}$ when using BPSK or QPSK modulation, and that hard coherent detection is about $2 \mathrm{~dB}$ worst than soft detection for these kinds of modulation. However, the main points that might be clarified is that differential binary phase shift keying (D-BPSK) 
exceeds direct detection on-off keying (DD-OOK) by $2.5 \mathrm{~dB}$ in power efficiency with robust codes, and that D-QPSK with low rate codes exceeds D-BPSK with high rate codes, with respect to power and bandwidth.

Shuo Shi, et al., in [23] proposed an effective and feasible method which is applied for demodulation of almost all LSNR BPSK signal types. This method combines chaotic theory with a control algorithm then compares with coventional methods such as the direct coherent demodulation method,which can only demodulate the signal with SNR greater than $0 \mathrm{~dB}$. The results show the prevalence of the proposed methodology and that the BER is associated with the sampling rate.

According to [3] and [24], a method is presented for demodulating QPSK signals after signal restoration which leads to low error rate and an increase in SNR. This method is based on chaotic synchronization. Chaotic synchronization refers to that two chaos systems with different initial parameters turn into the same state after a period of time with some synchronization control method such as SG (speed gradient) algorithm and the results indicate the chaotic method increases the immunity of the system against noise. With a comparison of chaotic synchronization and original coherent demodulation, the new technique improves the quality of detection particularly when the signal to noise ratio ranges from $-25 \mathrm{~dB}$ to $0 \mathrm{~dB}$. However, when $\mathrm{SNR}$ is less than $-25 \mathrm{~dB}$ with a noise bandwidth within $1 \mathrm{MHz}$, this technique cannot demodulate QPSK signals successfully.

M. C. Gursoy in [25] analysed the capacity of the MPSK system over fading and nonfading channels with hard detection in LSNR environments. The performance of fading channels is examined for coherent and non-coherent detections. Also, the spectral efficiency per energy of bit trade-off is analysed in spectral efficiency equal to zero.

Rahul, T. and Anant, S. presented an analysis for the basic bounds on detection performance in LSNR within the presence of noise uncertainty [18]. This analysis focuses on the initial transmissions of BPSK random information with non-pilot tones or coaching sequences.

Every detector has an SNR threshold below which it becomes impossible to detect signals within the noise uncertainty, and in the neighbourhood of this SNR wall the detection complexity approaches eternity.

P. Zhang et al. presented an analysis for energy efficiency and quality of the channel for OOK modulation with hard and soft decisions and made trade-offs between them [15]. They found that the non-coherent OOK modulation with hard decision is able to satisfy the Shannon limit. Besides, they provided analysis for the non-coherent channel of OOK modulation scheme within the LSNR to detect the minimum required SNR per bit with a highly reliable communications system. Additionally, they showed that channel investigations cannot be accessed at slow Rayleigh fading.

Tobias and Amos proved the capability of the discrete-time Gaussian channel in LSNR when its output is quantized with a one-bit quantizer [26]. A conventional symmetric threshold quantizer that is employed to reduce channel capacity by $2 / \pi$ usually causes an asymptotic power loss of approximately $2 \mathrm{~dB}$ and this loss is avoided entirely by using uneven threshold quantizers with corresponding asymmetric signal constellations.

D. F. Crouse et al. suggested a design that uses optimal memoryless relay sensor and investigated several channels, including random phase and fading channels, and apply different modulation schemes such as orthogonal frequency shift keying (OFSK) and OOK [27]. This method transmits signals only at nonzero energy with reliable communication.

A recursive algorithm is suggested to the symbol decision for UWB binary-PPM signals based on information theory standards which permits a reduction in the probability 
of bit error. This method can be used in case of no interframe interference [28]. It also has low cost when compared with the optimal symbol detector.

In [29], the performance of a category of phase detectors for carrier synchronization phaselocked loops (PLLs) in MPSK receivers has been analyzed in LPR systems. Naima et al. proposed an associate degree economical approach using a Track-Before-Detect (TBDF) filter for target detection and trailing within the LSNR conditions supported particle filtering, which incorporates un-thresholded information and a binary variable of the existence of the target within the estimated target state for two motion model [30].

Darin et al introduced a design and implementation a Quanta tracking (QT) algorithm. This method is able to to trace dim targets additionally that may tracked the LSNR [31]. One methodology was conjointly given to calculate SNR in step with Poisson's assumptions. However, the Poisson assumptions is that the distinction between the mean level in a pixel at the top of a "peak" and also the base, divided by the square root of the pixel variance.

Other related works concerned with the energy detectors in cognitive radio systems are given during this review. Cognitive radio may be a technique that permits users to investigate the spectrum to opportunistically transmit in obtainable frequency bands. Spectrum sensing is used to search about temporarily vacant frequency bands which can be utilized by illegal users [32].

The performance of the cognitive radio system depends on its ability to maximize the utilization of the free frequency band while not inflicting any interference to the primary user. Thus, the ability to detect the primary user and to avoid any wrong alarm is of paramount importance to such a system. For spectrum sensors supporting the energy detector in cognitive radios, these capabilities are affected by the threshold specified for noise signal discrimination. The traditional energy detectors of the fixed-threshold cognitive radio system might not be optimum under LSNR conditions wherever the performance of the fixed-threshold detection devices can differ considerably from the targeted performance measures [33].

In [33], a new perspective on the role of sensing time in determining the performance metrics in LSNR channels is investigated. This work proposes a method to set the threshold depending on the number of samples to enhance probability of detection and probability of false alarm. This proposed adaptive method is more appropriate for the changing operational requirements than those obtained by the fixed threshold energy detector. Similar approaches are also proposed in [34].

On the other hand, an analysis of the energy detection performance within LSNR channels is carried out, and a new method for probability of miss detection has been proposed in [35]. An optimum threshold criterion has been developed to reduce the overall error rate subject to the probability of false detecting. Closed form expressions for the optimum thresholds for the in non-fading channels and multipath fading channels were extracted in approximate form. Also, a method of combining diversity techniques and cooperative spectrum sensing is introduced to reduce the required sensing time or sampling rate to permit detection of signals in LSNR channels.

In [36] a new method for threshold detection is proposed to improve the performance of the radiometric detector in LSNR AWGN channels by reducing the probability of a complete detection error. The numerical results show an average improvement in performance of about 30\% from the traditional design in LSNR environment.

However, it is important to comprehend the basic limits to the detection with hard decision also the performance gained by soft detection can be determined and compared under the increasingly complex requirements. Driven by these concerns, the performance of the soft 
and hard decision is confirmed at LPR. An important work has been carried out in LSNR values where there is a difference of approximately $2 \mathrm{~dB}$ between decoding performance of soft and hard decisions in the range where 10-6< Probability of error $(\mathrm{Pe})<10-2$, and the capacity of an AWGN at LSNR values is equal to (1/ ln 2) SNR [37].

\section{CHANNEL CAPACITY}

Several studies have been carried out to evaluate the capacity of fading and AWGN channels under transmission of low power signals. A simple approximation methodology for the symmetrical capacity of Rayleigh channels with limited input data and ideal channel state information (CSI) was proposed in [38]. This approximation is extremely narrow in all SNR bands and is considered as a good estimation for the channel capacity.

R. Zouheir and S. A. Mohamed explained the capacity of Rayleigh and Nakagami-m flat fading channels with full channel state information (CSI) for the transmitter and receiver at asymptotically LSNR conditions [39]. Also, the CSI transmitter shows that a mono transmitter is suitable for realizing this rounding capability, which uses the power-out control system. Furthermore, the capacity for fading channels at LSNR environment was also studied in [40] and [41].

The studies presented in [42] and [43] proved that the capacity of these channels, within the broadband border, is approximately equivalent to that of the broadband AWGN nonfading channel; however the incoming signals, like MFSK, are extremely burst within time or frequency domain.

Also, it is demonstrated that if the speed of signals at each frequency and time is limited, the capacity of these broadband channels would be severely restricted. Specifically, the energy needed for each bit is close to infinity [44].

Vignesh and Bruce in [45] provided a method for the analysis of flat-fading channels under maximum and average energy constraints. The lower and upper limits are derived to capacity per unit time to separate time channels in low SNR environment.

Lizhong Zheng et al. explained some significant constraints within the coherent and noncoherent fading channels including peak signaling requirements and the resulting spectral efficiency, and how these factors limit capacity when SNR tends to zero [46].

In LSNR (MIMO) techniques, conventional methods used in high-SNR systems are highly dependent on channel coherence to reduce interference. It is also clear that more energy efficiency can be achieved by adapting the multi-antenna encoder with channel conditions [47].

Other related work that presented by Shashi and Lizhong in [48] proposed a design based on the training principle for such a channel as the flat fading Rayleigh channel in LSNR with full CSI in the transceiver. The proposed scheme is also used to find the channel capacity of the CSI case of the noisy transmitter. Zouheir and Mohamed Alouini in [49] analyzed the throughput capacity area of the Gaussian multiple-access (MAC) fading channel with an ideal CSI in low power system transceivers. Also, they proposed a simple on-off scheme, computed its possible rate and characterized a class of fading.

Muriel and Jinfeng in [50] introduced an analysis of the capacity bounds using network equivalence theory and a multiple-layer of frequency shift keying (FSK) scheme within the LSNR regime. Also, they investigated and characterized the low and high power transmission of a network within multiple relays in the broadband system through memory separate fading channels, without having a priori channel standing an information. 
Bitan et al. in [51] proposed two novel approximations for the ergodic capacity in LSNR channels that are general and need few moments only. This approximation uses channel moments that does not have to be updated for every distinct SNR value. The results illustrate the high accuracy of the proposed estimate amongst several other estimates. Additionally, the results showed that estimate of the proposed methodology is used efficiently to search for several kinds of wireless links. The high accuracy of approximation was achieved for several combinations of channels.

Fatma Benkhelifa et al. in [52] presented an analysis of the ergodic capacity of the MRC Rician fading channel with full CSI in transmitter and receiver in the LSNR regime. One main result found is that one-bit CSI feedback at the transmitter was sufficient to achieve this capacity using the power-off control system.

Srinivasan and Varanasi [53] presented two significant problems to meet high-reliability communications through the non-coherent MIMO technology with the Rayleigh fading channels in LSNR systems. In each Peak-Power-to-Average-Power Ratio (PPAPR) restriction, the optimum constellations are acquired in closed form rather than non-convex optimization.

The LSNR Asymptotics of block fading channels for the capability of MIMO channels with no facet information has been introduced in [54]. However, the peak limits are obligatory on coefficients in an exceedingly given signal illustration, rather than difficult restrictions on the signals sent.

David T. and Pramod V. in [55] illustrated that the cutoff capacity might be a restricted part of the capacity of the AWGN channel. Also they showed that, in LSNR conditions, the capacity of the fading channel is approximately equal to the capacity of the AWGN without fading [55]:

$$
C=E\left[\log _{2}\left(1+|h|^{2} S N R\right)\right] \approx E\left[|h|^{2} S N R\right] \log _{2} e=S N R \log _{2} \approx C_{\text {awgn }}
$$

Where:

$\mathrm{C}_{\text {awgn }}$ is the capacity of the AWGN channel evaluated in bits/symbol.

$\mathrm{h}$ is the channel gain.

$\mathrm{E}$ is the Mean value.

On the other hand, at high SNR [55]:

$$
C \approx E\left[\log _{2}\left(|h|^{2} S N R\right)\right]=\log _{2} S N R+E\left[\log _{2}\left(|h|^{2}\right)\right] \approx C_{\text {awgn }}+E\left[\log _{2}\left(|h|^{2}\right)\right]
$$

Furthermore, it was shown that the quantity of energy needed at the infinite spectrum limit for the Rayleigh-fading channel to reliably transmit one data bit is around $E_{b} / N_{o}=$ $1.59 \mathrm{~dB}$, which is an equivalent limit to the AWGN channels. This tremendous outcome is equal to [46]:

$$
\lim _{S N R \rightarrow 0} \frac{C_{\text {fading }}(\mathrm{SNR})}{\mathrm{SNR}}=\lim _{S N R \rightarrow 0} \frac{\mathrm{C}_{\mathrm{awgn}}(\mathrm{SNR})}{\mathrm{SNR}}=1
$$

Also, it carries no matter whether CSI is instantaneously available on the receiver or not, although it assumes no CSI in the transmitter.

\section{LSNR ESTIMATION TECHNIQUES}

An acceptable SNR estimation technique in communication systems is needed since various receiver optimisation algorithms need SNR information to optimum performance if the SNR is time varying. The performance of such communication systems can be improved if correct SNR estimators are utilized [56]. There are several studies about SNR 
estimation, where many communication systems need a precise and low bias estimation of the SNR for optimum performance. In modern wireless networks, every modulation and coding method is designed for transmitting information to suit channel capacity for the given SNR [57]. Also, SNR data are extremely necessary for detection and decoding techniques that represent the most recent for wireless technology [58]. The SNR estimation technique is commonly demanded for several applications like power management, equalization, delivery, and effective resource allocation [59].

However, the SNR factor represents a valuable limitation in many communications receivers, and for them, the proper operations depend heavily on the correct estimate of the SNR ratio. It is also noted that SNR estimation is required to run the system at higher data rates [60]. A method was proposed in [60] to estimate the SNR value for QPSK scheme that is dependent upon the second and fourth moments without any iterative process. The reliable range of the estimated SNR to a minus-decibel area of SNR extends from -15 to 20 $\mathrm{dB}$ and therefore the results can even apply to 8-PSK by simple modification. A systematic study on SNR estimation is performed with a new algorithm for BPSK signals is proposed for SNR estimation, and this algorithm is based on a frequency solution to estimate the probability of maximum SNR capacity [61]. The results show that there is less bias and normalized MSE than other techniques, and the useful range extends to negative SNRs. In [62], a design and implementation of an estimator for M-ary phase-shift keying (MPSK) modulation is presented depending upon the anticipation of the statistical characteristics of the received signals with a limited observation length. Results showed that the proposed estimator could be implemented within the FPGA platform. Also, it has the best performance among some other examined estimators with a highly accurate and low bias SNR estimation. The proposed estimator is exceptionally an exact in the SNR range from $10 \mathrm{~dB}$ to $20 \mathrm{~dB}$ and may be widely utilized in several fields, like adaptive transmission, turbo decoding, power management, and so on.

In [63], a novel estimation technique for BPSK and QPSK signals is proposed based on non-data aided (NDA) technique. A performance comparison was made via the suggested method and the other NDA estimators. The results showed that the proposed technique is more accurate than others, especially in LSNR values that make it useful for practical applications in the complex AWGN channel. In [64], another NDA SNR estimation technique is described for non-equiprobable and equiprobable signal transmission using non-coherent detection of OOK signals.

Finally, some concluding remarks focusing on SNR estimation are given in [65], and [66] where two SNR estimator methods are proposed which maintain the accuracy of the estimation. The error correction techniques also use SNR to determine soft decision-making possibilities and probability ratios easily.

\section{LSNR CODING TECHNIQUES}

Optimal coding adaptation is conferred during this work. However, several applications For instance, in cellular-based machine-to-machine communications, wherever an oversized variety of low-power devices randomly transmit their information to a single base station, several devices could also be in deep fade because of their constant movement. For this kind of network, dedicating resources, like bandwidth or even relays, to the devices is not efficient because of the random bursty nature of the transmission [9]. This will include the study of effective transmission techniques for LSNR communications systems, wherever transmission capacity or cooperative techniques cannot be exaggerated, and that channel 
coding is the sole possibility [9]. Coding techniques like low-density parity-check (LDPC) code, turbo code, and the alternative advanced coding method permit the utilization of LSNR communication systems [8].

In [67], a valid coding and modulation for MIMO free-space optical system for M-ary PPM is described based on LDPC and multilevel coding. The suggested method gives incredible coding gain within the regime of robust atmospherical turbulence. However, the M-ary PPM is an attractive power-efficient modulation scheme for Free-Space Optical (FSO) links.

Mahyar and Sarah in [9] explored the design of Raptor codes where the coding efficiency and decoding complexity were considered when optimizing the degree distribution, Also they proposed the upper limit on the extreme rated power efficiency of the Raptor code. Results show that in very low SNRs, the Raptor code with the designed distribution functions is closely approaching the AWGN channel capacity.

G. Jan et al. Proposed an efficient LSNR early termination (LSNR ET) design, which is verified by employing a typical transmission with the long term evolution (LTE) mobile standard [68][69]. This scheme is extremely efficient if the typical range of iterations for a turbo decoder is comparatively little. For typical mobile transmission channels, represented by the typical urban (TU) channel model, LSNR ET has become a vital part to reduce the complexity of the turbo decoding process in LTE systems.

Samah Ghanem presented a new look of a piggybacking for network cryptography where robust supply inputs piggyback the weaker ones, a theme necessary and comfortable to attain the cut-set limited at high to low SNR (H/LSNR) systems, a replacement that is optimum in operation for the multi-hop amplify and forward (AF) networks [70].

\section{THE SPECTRAL EFFICIENCY}

In LPR systems, the energy per bit to the noise power spectral density ratio is the main concept for capturing the trade-off between bandwidth and power. Suppose, in the absence of loss of generality, which complicated symbol take a $(1-\mathrm{s} \times 1-\mathrm{Hz})$ the time and frequency slot, as well the most accomplishable spectral efficiency as a function of the energy per bit to noise power spectral density ratio $\mathrm{C}(\mathrm{Eb} / \mathrm{No})$ may be obtained from the Shannon capacity [71]:

$$
\mathrm{C}(\mathrm{Eb} / \mathrm{No})=\mathrm{C}(\mathrm{SNR}) \text { bits } / \mathrm{s} / \mathrm{Hz}
$$

where:

$$
\mathrm{Eb} / \mathrm{No}=\mathrm{SNR} / \mathrm{C}(\mathrm{SNR})
$$

Power was normalized slightly to noise strength. However, the total description of the spectral efficiency of the function of bit per energy through Shannon's capacity is either unknown or numerically calculated for several attenuation channels whose achievements do not seem to be legendary in the receiver. Thus, one must use approximation strategies to check the spectral efficiency of a large variety of attenuating channels.

In [71], an analysis is presented to PSK's energy efficiency when get combined with OOK for transmission through non-coherent Rician channels. If there is a fixed peak, the results indicate that the OOK signal is given at level with peak, which achieve the minimum energy per bit. Other work by Wenyi Zhang and J. Nicholas Laneman investigates the achievable rates of PSK on non-coherent Rayleigh fading channels [72].

Mustafa C., et al., in [73] demonstrated the spectral efficiency of each bit of power in the LPR by getting a minimum of energy per bit and broadband slope that may be 
accomplished through the pure OOK signal. The results indicated that OOK is the best signal within the LPR system to reach the minimum bit power and broadband slope, whereas the OOK and FSK signals are to be combined to enhance energy efficiency. Jesse and Jan in [74] proposed a new technique to perform energy detection of a ULP-OOK system. This system uses an oversampling and digital balance to scale back the surplus noise addition in the channel. Additionally, the oversampling technique provides additional accuracy in time for synchronization and clock extraction for the remainder of the radio system.

In order to reduce the energy consumed by the transceiver it is observed in [75] a design to enhance SNR for producing one bit of information without error. Results showed that SNR is optimized with minimum energy consumption at completely different transmission distances and different channel statistics.

\section{CHALLENGES AND REQUIREMENT}

After completing the comprehensive survey of previous works in the field of the LSNR Communication Systems, the main requirement and challenges of general communication systems and communication systems in LSNR environments are listed below in Table 1. However, this survey guides the reader through the extensive open literature Research

TABLE 1. MAIN REQUiREMENT OF COMMUNICATION SYSTEMS AND LSNR COMMUNICATION SYSTEMS

\begin{tabular}{cccc}
\hline Factor & $\begin{array}{c}\text { Ideal Communication } \\
\text { system }\end{array}$ & $\begin{array}{c}\text { Normal communication } \\
\text { system }\end{array}$ & $\begin{array}{c}\text { LSNR } \\
\text { communication system }\end{array}$ \\
\hline Required power (Eb/No) & Low & High & $\sqrt{ }$ Low \\
Transmission rate ( R) & High & High & Low $\uparrow$ \\
System utilization ( U) & High & High & Mid/low $\uparrow$ \\
BER (Pe) & Low & Low & Mid $\downarrow$ \\
Probability of detection(Pd) & High & High & Mid/low $\uparrow$ \\
Bandwidth (W) & low & Low & High/mid $\downarrow$ \\
System complexity ( Cx) & low & Mid & Mid $\downarrow$ \\
\hline
\end{tabular}

Note: The symbols of the applicability in table $1: \sqrt{ }:$ Applicable.

\section{CONCLUSIONS}

In this work, a survey for digital communications has been conducted for low SNR systems and for a wide range of applications. As a result of this review, it is important to confirm the availability of communication in the high noise area when designing low-power systems provided that appropriate modulation and detection techniques are used. Several methods can be considered to enhance user performance as well as network performance and to improve energy efficiency as mentioned in the reviewed works. The OOK and BPSK modulation schemes are preferred for sending data in LSNR regimes. For a suitable trade-off between performance and complexity, on-off keying is a promising candidate to make low-rate and high-reliability communications possible in ULP communication systems. 


\section{REFERENCES}

[1] R.W. Middlestead, "Digital communications with emphasis on data modems: Theory, analysis, design, simulation, testing, and applications", John Wiley \& Sons. , 2017.

[2] D. Kumar, M. Khaliluzzaman, and M. Faisal, "Performance Analysis of Digital Communication Systems in Presence of AWGN Implementing Filter Technique". International Journal of Computer Applications, 131(18), pp.5-10. 2015.

[3] Q. Ren, S. Shi, , D. Li, and X. Gu, "Demodulation of Low SNR QPSK Signal Based on Chaotic Synchronization". IEEE Sixth International Conference on Instrumentation \& Measurement, Computer, Communication and Control (IMCCC), pp. 609-613. July 2016.

[4] W. Jørgen Falch, "Develop of a proof of concept for the utilization of data for an optimal and controllable indoor environment at the DTU Library Living Lab". Technical University of Denmark, Department of Civil Engineering, 2016.

[5] M. Leba, S. Riurean, and A. Lonica, LiFi-The path to a new way of communication. In 2017 12th Iberian Conference on Information Systems and Technologies (CISTI) (pp. 1-6). IEEE. 2017.

[6] F. Passerini, and A.M. Tonello, "Smart grid monitoring using power line modems: Anomaly detection and localization". IEEE Transactions on Smart Grid. 2019.

[7] P. Konstantinos and C. Luca, " On Adaptive Modulation for low SNR Underwater Acoustic Communications", OCEANS 2018 MTS/IEEE Charleston, pp. 1-6, October 2018.

[8] J.Yang, L. Guangxia, Z.Hongpeng , and Z. Xiaokai, “ Design of Synchronous Structure of Receiver at Very Low SNR". First International Conference on Electronics Instrumentation \& Information Systems. 2017.

[9] M. Shirvanimoghaddam, and S. Johnson, "Raptor codes in the low SNR regime". IEEE Transactions on Communications, vol. 64, pp.4449-4460, 2016.

[10] G. Zijun , L. Cheng and J. Fan, "Channel Estimation for Sparse Massive MIMO Channels in Low SNR Regime". IEEE Transactions on Cognitive Communications and Networking, vol. 4, Dec. 2018

[11] R. Hu, and Y. Wang, “A first arrival detection method for low SNR microseismic signal”. Acta Geophysica, vol. 66, pp.945-957, 2018.

[12] A. B. Naicker and M. Tamilarasi, "UWB Transmitter using OOK Modulation Technique in 45 nm Technology". International Conference on Innovations in Information Embedded and Communication Systems (ICIIECS). 2017.

[13] F. Rosas, and C. Oberli, “ Modulation and SNR optimization for achieving energy-efficient communications over short-range fading channels". IEEE Transactions on Wireless Communications, vol. 11, pp.4286-4295. , 2012.

[14] I. Kianpour, B. Hussain, and V.G. Tavares, “ A complementary LC-tank based IR-UWB pulse generator for BPSK modulation ”. IEEE East-West Design \& Test Symposium (EWDTS), pp. 1-4. September 2017.

[15] P. Zhang, F.M. Willems, and L. Huang, " Capacity investigation of on-off keying in noncoherent channel settings at low SNR”. Transactions on Emerging Telecommunications Technologies, vol. 26, pp.1235-1250. 2015.

[16] S. Verdú, "Spectral efficiency in the wideband regime". IEEE Transactions on Information Theory, vol 48, pp.13191343. , 2002.

[17] E. Leitinger, B.C. Geiger, and K. Witrisal, " Capacity and capacity-achieving input distribution of the energy detector", IEEE International Conference on Ultra-Wideband, pp. 57-61. September 2012.

[18] R. Tandra, and A.Sahai, "Fundamental limits on detection in low SNR under noise uncertainty". IEEE International Conference on Wireless Networks, Communications and Mobile Computing, vol. 1, pp. 464-469, June 2005.

[19] W.Zhang, and J.N.Laneman, "Benefits of spatial correlation for multi-antenna non-coherent communication over fading channels at low SNR". IEEE transactions on wireless communications, vol. 6, pp.887-896. 2007.

[20] X.Zhu , T. Wang, Y. Bao, F.Hu, and S. Li, "Signal Detection in Generalized Gaussian Distribution Noise With Nakagami Fading Channel”. IEEE Access, 7, pp.23120-23126. , 2019.

[21] X. Luo, and G.B. Giannakis, "Energy-constrained optimal quantization for wireless sensor networks". EURASIP Journal on Advances in Signal Processing, p.73. 2008.

[22] G. Kramer, A. Ashikhmin, A.J. van Wijngaarden, and X.Wei, "Spectral efficiency of coded phase-shift keying for fiber-optic communication". Journal of lightwave technology, vol. 21, p.2438, 2003.

[23] S. Shi, J. Ji, and X. Gu, "Weak BPSK signal demodulation by chaotic synchronization method". IEEE Fifth International Conference on Computing, Communications and Networking Technologies (ICCCNT), pp. 1-4. July 2014.

[24] X. Xian, and J. Liu, "Application of Chaos Theory in Incomplete Randomized Financial Analysis", Korea, 2(1.6), pp.3-5. 2019.

[25] M.C. Gursoy , "On the low-SNR capacity of phase-shift keying with hard-decision detection". IEEE International Symposium on Information Theory, pp. 166-170. June 2007.

[26] T. Koch, and A. Lapidoth , "At low SNR, asymmetric quantizers are better". IEEE Transactions on Information Theory, vol. 59, pp. 5421-5445, 2013.

[27] D.F. Crouse, C.R. Berger, S. Zhou, and P.Willett, " Optimal memoryless relays with noncoherent modulation”. IEEE Transactions on Signal Processing, vol. 56, pp. 5962-5975, 2008.

[28] J.A. López-Salcedo, and G. Vázquez, "Detection of PPM-UWB random signals". IEEE Transactions on Signal Processing, vol. 56, pp. 2003-2016, 2008.

[29] W. Jiang, and Y. Cui, "Performance analysis of MPSK phase detectors for carrier synchronization PLLs at low SNRs". IEEE Communications Letters, vol. 18, pp..2133-2136. 2014.

[30] N. Amrouche, A. Khenchaf, and D. Berkani , "Detection and tracking targets under low SNR". IEEE International Conference on Industrial Technology (ICIT), pp. 992-996. March 2017. 
[31] D.T. Dunham, P.K. Willett, and T.L. Ogle, “ Quanta Tracking Algorithm for Low SNR Targets: How Low Can it Go?". IEEE 21st International Conference on Information Fusion (FUSION), pp. 1582-1588, July 2018.

[32] B. S Fabrício, " Performance of Cognitive Spectrum Sensing Based on Energy Detector in Fading Channels", Procedia Computer Science, 65, pp. 140 - 147, 2015.

[33] R.N. Prashob, A.P. Vinod, and A.K. Krishna, "An adaptive threshold based energy detector for spectrum sensing in cognitive radios at low SNR". IEEE International Conference on Communication Systems, pp. 574-578, November 2010.

[34] R. Gao, X.Chen, Z. Li, M.Yang, and H. Chang, “Efficient Spectrum Sensing Scheme under Low SNR”. IEEE International Conference on Computer and Information Technology, pp. 856-859, September 2014.

[35] S. Atapattu, C. Tellambura, H. Jiang, and N. Rajatheva, , " Unified analysis of low-SNR energy detection and threshold selection ". IEEE Transactions on vehicular technology, vol. 64, pp.5006-5019. 2014.

[36] D. Raman, N.P.Singh, and M.K. Dhaka, “ Low SNR radiometric detection in cognitive radio”. IEEE International Conference on Signal Processing and Integrated Networks (SPIN) ,pp. 322-326, February 2014.

[37] U. Madhow, " Fundamentals of digital communication”. Cambridge University Press. , 2008

[38] S.B. Slimane, "Approximation to the symmetric capacity of Rayleigh fading channels with multi-level signals". IEEE communications letters, vol. 10, pp.129-131. 2006.

[39] Z. Rezki, and M.S. Alouini, " On the capacity of Nakagami-m fading channels with full channel state information at low SNR”. IEEE Wireless Communications Letters, vol. 1, pp.253-256, 2012.

[40] T. Li, X. Jin, and O.M. Collins, "Approaching capacity on correlated fading channels with unknown state". IEEE Proceedings. International Symposium on Information Theory (ISIT), pp. 520-524, September 2005.

[41] S.N. Sur, R. Bera, and B. Maji, "Phased-MIMO Radar in Low SNR Regime". In Advances in Communication, Devices and Networking, pp. 357-362. Springer, Singapore. 2019.

[42] I.E. Telatar, and D.N.C.Tse, "Capacity and mutual information of wideband multipath fading channels". IEEE transactions on information theory, vol 46, pp.1384-1400., 2000.

[43] G. Durisi, H. Bolcskei, and S. Shamai " Capacity of underspread WSSUS fading channels in the wideband regime". IEEE International Symposium on Information Theory pp. 1500-1504. July 2006.

[44] M. Médard, and R.G. Gallager, “ Bandwidth scaling for fading multipath channels”. IEEE Transactions on Information Theory, vol. 48, pp.840-852. 2002.

[45] V. Sethuraman, and B. Hajek , "Low SNR capacity of fading channels with peak and average power constraints". In 2006 IEEE International Symposium on Information Theory, pp. 689-693. July 2006.

[46] L. Zheng, N.C. David, and M. Médard , "Channel coherence in the low-SNR regime". IEEE Transactions on Information Theory, vol. 53, pp.976-997. 2007.

[47] G. Miao , N. Himayat , Y. Li, and A. Swami , “ Cross-layer optimization for energy efficient wireless communications: a survey". Wireless Communications and Mobile Computing, vol. 9, pp.529-542. 2009.

[48] S.Borade, and L. Zheng , "Wideband fading channels with feedback”. Institute of Electrical and Electronics Engineers. 2010.

[49] R. Zouheir and A. Mohamed-S. , " On the Capacity of Multiple Access and Broadcast Fading Channels with Full Channel State Information at Low SNR". IEEE Transactions on Wireless Communications, pp.464 - 475. 2014.

[50] M.Muriel and D. Jinmen, "Low SNR - When Only Decoding Will Do". IEEE Global Conference on Signal and Information Processing (GlobalSIP). 2014

[51] B.Banerjee, A.A. Al Haija, C. Tellambura, and H.A.Suraweera, " Simple and accurate low SNR ergodic capacity approximations". IEEE Communications Letters, vol. 22, pp.356-359. , 2017.

[52] F. Benkhelifa, Z. Rezki, and M.S. Alouini, " On the low SNR capacity of maximum ratio combining over Rician fading channels with full channel state information ". IEEE Wireless Communications Letters, 2(2), pp.191-194. , 2013.

[53] S.G. Srinivasan, and M.K Varanasi, “ Optimal constellations for the low-SNR noncoherent MIMO block Rayleighfading channel ". IEEE Transactions on Information Theory, vol. 55, pp.776-796. 2009.

[54] C. Rao, and B.Hassibi, " Analysis of multiple-antenna wireless links at low SNR". IEEE Transactions on Information Theory, vol. 50, pp.2123-2130, 2004.

[55] D.Tse, and P. Viswanath, “Fundamentals of wireless communication” Cambridge University Press. 2005.

[56] N.S. Kamel, and V. Jeoti, "A Linear Prediction Based Estimation of Signal to Noise Ratio in AWGN Channel”. ETRI journal, 29(5), pp.607-613, 2007.

[57] B. Paul, A.R. Chiriyath and D.W.Bliss, " Survey of RF communications and sensing convergence research". IEEE Access, 5, pp.252-270. , 2016.

[58] L. Hanzo, P. Woodard, P. Robertson, "Turbo decoding and detection for wireless applications". Proceedings of the IEEE, vol. 95, pp. 1178-200, 2007.

[59] N. Beaulieu, A. Toms, D. Pauluzzi, "Comparison of four SNR estimators for QPSK modulation". IEEE Communication Letters, vol. 4, pp.43-45, Feberory. 2000.

[60] G. Ren, Y. Chang, and, H.Zhang, "A new SNR's estimator for QPSK modulations in an AWGN channel”. IEEE Transactions on Circuits and Systems II: Express Briefs, vol. 52, pp.336-338, 2005.

[61] B. Li, , R. DiFazio, and A. Zeira, " $\backslash$ A low bias algorithm to estimate negative SNRs in an AWGN channel". IEEE Communications Letters, vol. 6, pp.469-471. , Dec 2002.

[62] C. Gong, B. Zhang, A. Liu, and D. Guo, "A Highly Accurate and Low Bias SNR Estimator: Algorithm and Implementation". Radioengineering, vol. 20, 2011.

[63] A. Ijaz, A.B. Awoseyila, and B.G. Evans, "Improved SNR estimation for BPSK and QPSK signals". Electronics Letters, vol. 45, pp.858-859. 2009. 
[64] V. Sridharan, N.F. Kiyani, H. Nikookar, J.H. van den Heuvel, and G. Dolmans, “NDA SNR estimation techniques for non-equiprobable signaling using non-coherent OOK receivers". IEEE International Conference on Communications (ICC), pp. 5559-5563, June 2013.

[65] M. Zhou, H.Yang, J. Zhu, and J. Shi, , "Low SNR signal time-frequency analyzing method". IEEE International Conference on Image Analysis and Signal Processing pp. 21-25, October 2011.

[66] F. Harris, and C. Dick, " SNR estimation techniques for low SNR signals". IEEE The 15th International Symposium on Wireless Personal Multimedia Communications, pp. 276-280,, September 2012.

[67] I.B. Djordjevic, B. Vasic, and M.A. Neifeld, "Multilevel coding in free-space optical MIMO transmission with Q-ary PPM over the atmospheric turbulence channel". IEEE photonics technology letters, vol. 18, pp.1491-1493, 2006.

[68] J. Geldmacher, K. Hueske, J. Götze, and M. Kosakowski, "Hard decision based low SNR early termination for LTE Turbo decoding". IEEE 8th International Symposium on Wireless Communication Systems, pp. 26-30, November,2011.

[69] S.A.Lonkar, K.T.V. Reddy, and S.P.Singhania, " Novel Cell Search Method in Long-Term Evolution System ". In Proceeding of the Second International Conference on Microelectronics, Computing \& Communication Systems (MCCS 2017) (pp. 623-631). Springer, Singapore. , 2019.

[70] S.A. Ghanem, "Piggybacking Codes for Network Coding: The High/Low SNR Regime". GLOBECOM IEEE Global Communications Conference, pp. 1-6, December 2017.

[71] M.C. Gursoy, H.V. Poor, and S. Verdú , "Noncoherent Rician fading Channel-part II: spectral efficiency in the lowpower regime ". IEEE Transactions on Wireless Communications, vol.5, pp.2207-2221, 2005.

[72] W. Zhang, and J.N. Laneman, " How good is PSK for peak-limited fading channels in the low-SNR regime? ”. IEEE transactions on information theory, vol. 53, p.236. , 2007.

[73] M.C. Gursoy, H.V. Poor, and S. Verdú, "On-off frequency-shift keying for wideband fading channels". EURASIP Journal on wireless communications and networking, vol, 2006, p.098564, 2006.

[74] J. Richmond, and J. Rabaey, "Digital energy detection for OOK demodulation in ultra-low power radios". IEEE International Symposium of Circuits and Systems (ISCAS), pp. 1808-1811, May 2011.

[75] F. Rosas, and C. Oberli, "Modulation and SNR optimization for achieving energy-efficient communications over short-range fading channels". IEEE Transactions on Wireless Communications, vol. 11, pp.4286-4295, 2012. 